\title{
Colecistectomia videolaparoscópica em vesícula biliar duplicada: relato de caso
}

\author{
Laparoscopic cholecystectomy in duplicated gallbladder: case report
}

Colecistectomía Videolaparoscópica en vesícula biliar duplicada: reporte de un caso

Eduardo Augusto Pinheiro Pimenta ${ }^{1 *}$, Thais Regina Moreira Printes ${ }^{1}$, Nayandra Sales Bendaham², Juan Eduardo Rios Rodriguez ${ }^{2}$, Nastassja Sales Bendaham², Elizeu Rodrigues Matos ${ }^{2}$, Suany Serudo Meirelis² ${ }^{2}$ Danielle Alcântara Barbosa ${ }^{1}$.

\section{RESUMO}

Objetivos: Apresentar um paciente com variação anatômica na vesícula biliar do tipo vesica fellea divisa, que, apesar de congênita, só foi evidenciada na quarta década de vida após um fator desencadeante sintomático. Relato de caso: Paciente masculino, 41 anos, relatava dor difusa de leve intensidade em hipocôndrio direito aliviada com uso de sintomáticos. Negava qualquer manifestação colestática. Referiu início do sintoma há dois anos, quando apresentou episódio álgico de maior intensidade e com irradiação para tórax, necessitando de internação por quatro dias. À exceção da dor em hipocôndrio direito na palpação profunda, o exame físico não mostrou alterações. A ultrassonografia realizada sugeriu presença de vesícula biliar bilobulada com presença de múltiplos cálculos em seu interior. Considerações finais: A importância clínica do caso está associada com o desenvolvimento de colelitíase, uma vez que a condição anatômica promove uma drenagem inadequada da bile e também com um maior risco de complicações perioperatórias, sendo esta condição considerada uma das mais associadas à lesão iatrogênica do ducto biliar durante realização de colecistectomias. Conclui-se que a conduta mais efetiva após a sua identificação é a retirada total do órgão, de modo a evitar reoperações, tendo em vista o mau prognóstico advindo da anomalia.

Palavras chave: Colecistectomia, Colelitíase, Vesícula Biliar, Anormalidades Congênitas.

\begin{abstract}
Objectives: To present a patient with anatomic variation in the gallbladder type vesica fellea divisa, which, although congenital, was only evidenced in the fourth decade of life after a symptomatic triggering factor. Case Report: A 41-year-old male patient reported diffuse mild pain in right hypochondrium relieved with pain relieving medications. He denied any symptoms of cholestasis. He referred to the onset of the symptom two years ago, when he presented a pain episode of greater intensity and with irradiation to the chest, requiring hospitalization for four days. With the exception of right hypochondrium pain on deep palpation, the physical examination showed no changes. Ultrasound performed suggested bilobular gallbladder with multiple gallstones in its interior. Final considerations: The clinical importance of the case is associated with the development of cholelithiasis, since the anatomical condition promotes an inadequate drainage of bile and also with a greater risk of perioperative complications, being this condition considered one of the most associated to the iatrogenic injury of the bile duct during cholecystectomy. It is concluded that the most effective conduct after its identification is the total removal of the organ, in order to avoid reoperations, due to the bad prognosis coming from the anomaly.
\end{abstract}

Keywords: Cholecystectomy, Cholelithiasis, Gallbladder, Congenital abnormalities.

${ }^{1}$ Hospital Universitário Getúlio Vargas, Manaus-AM. *E-mail: edu_med82@hotmail.com

${ }^{2}$ Universidade Federal do Amazonas (UFAM), Manaus-AM.

SUBMETIDO EM: $3 / 2019$

ACEITO EM: 4/2019

PUBLICADO EM: 7/2019

REAS/EJCH | Vol. 11(10)| e696 | DOI: https://doi.org/10.25248/reas.e696.2019 Página 1 de 5 


\section{RESUMEN}

Objetivos: Presentar un paciente con variación anatómica en la vesícula biliar del tipo vesica fellea divisa, que, a pesar de congénita, sólo fue evidenciada en la cuarta década de vida después de un factor desencadenante sintomático. Relato de caso: Paciente masculino, 41 años, relataba dolor difuso de leve intensidad en hipocondrio derecho aliviada con uso de sintomáticos. Negaba cualquier manifestación colestática. A excepción del dolor en hipocondrio derecho en la palpación profunda, el examen físico no mostró alteraciones. Refirió el síntoma temprano hace dos años, cuando presentó episodio álgico de mayor intensidad e irradiación para el pecho, que requirió hospitalización durante cuatro días. La ultrasonografía realizada sugirió presencia de vesícula biliar bilobulada con presencia de múltiples cálculos en su interior. Consideraciones finales: Su importancia clínica está asociada con el desarrollo de colelitiasis, ya que la condición anatómica promueve un drenaje inadecuado de la bilis y un mayor riesgo de complicaciones perioperatorias, siendo esta condición considerada una de las más asociadas a la lesión iatrogénica del conducto biliar durante la realización de colecistectomías. La conducta más efectiva después de su identificación es la retirada total del órgano, para evitar reoperaciones, teniendo en vista el mal pronóstico que viene de la anomalía.

Palabras clave: Colecistectomía, Colelitiasis, Vesícula Biliar, Anomalías Congénitas.

\section{INTRODUÇÃO}

A vesícula biliar tem grande relação com uma multiplicidade de variações anatômicas. A vesícula biliar duplicada é uma anomalia congênita rara, com incidência de 1: 4000 nascimentos (DESOULNEUX G et al., 2009; PINA LF et al., 2018). Apesar de rara, é uma condição que vem sendo descrita há muito tempo (PINA LF et al., 2018).

Em humanos, tem como característica predominante uma vesícula biliar dupla, com dois ductos císticos, cuja vesícula acessória surge como crescimento secundário do ducto cístico ou do ducto colédoco (BOYDEN EA, 1926).

Não há sintomas especificamente atribuídos a presença desta condição em um indivíduo. Logo, a descoberta desse achado anatômico se dá, geralmente, de modo incidental durante realização de exames de imagem, procedimentos cirúrgicos ou autópsias ou quando se tornam sintomáticos devido à presença de alguma outra condição de cunho patológico (ROMERO EXA et al., 2018; SHIBA H et al., 2014).

Mais raramente, porém, já foi relatada associação a condições mais graves, como anormalidades nos ductos biliares e/ou artéria hepática, malformações cardíacas, rins policísticos e inúmeras deformidades musculoesqueléticas (ELORZA JD et al., 2009; HARLAFTIS N et al., 1977).

Já foram descritos casos de inúmeras doenças da vesícula biliar em pacientes dotados desta variação anatômica, como coletitíase (a mais descrita), colecistite (tanto aguda quanto crônica), fístulas colecistocólicas e colecistocoledocianas, papilomas e carcinomas (adenocarcinoma e adenomiomas) (DESOULNEUX G, et al., 2009; ROMERO EXA et al., 2018; SHIBA H et al., 2014).

Contudo, a presença da duplicação da vesícula biliar não é fator independente para produção de maior predisposição ao desenvolvimento de tais doenças; assim, o aparecimento dessas patologias em portadores da variação em questão obedece aos mesmos fatores de risco e propensões encontradas naqueles com vesícula biliar única e apresentam a mesma sintomatologia (COELHO JCU et al., 2003; LOBO EJ et al., 2000). No que se refere às patologias de vesícula biliar cuja abordagem seja cirúrgica, a compreensão desta condição se faz necessária para que haja adequado planejamento do procedimento.

Assim, sua importância clínica se faz presente devido à associação dessa anomalia a um maior risco de complicações perioperatórias, como a lesão iatrogênica do ducto biliar durante realização de colecistectomias. Dada essa informação, fica evidente a necessidade de se realizar o diagnóstico pré-operatório dessa condição 
de modo a se evitar complicações perioperatórias e/ou execução de repetidas laparotomias. Também é reconhecida a relação da duplicação da vesícula com o desenvolvimento de colelitíase, uma vez que a condição anatômica promove uma drenagem inadequada da bile (PILLAY Y, 2015), sendo mais comum a presença de cálculo no interior de apenas uma das duas vesículas (ELORZA JD et al., 2009). Mesmo nesse caso, é recomendada a retirada completa de ambas as vesículas.

A realização de exames de imagem, em especial a ultrassonografia, auxilia tanto na detecção dessa variação anatômica, permitindo que haja uma diminuição de lesão iatrogênica às estruturas manipuladas durante um procedimento de colecistectomia, quanto na exclusão de entidades que possam mimetizar essa variação anatômica, como um cisto de ducto colédoco ou um divertículo de vesícula biliar (GOCMEN R e YESILKAYA Y, 2012).

\section{RELATO DE CASO}

Paciente masculino, 41 anos, relatava dor difusa de leve intensidade em flanco direito aliviada com uso de sintomáticos. Negava qualquer manifestação colestática. Referia início do sintoma há dois anos, quando apresentou episódio álgico de maior intensidade e com irradiação para tórax, necessitando de internação por 4 dias em serviço de atendimento de urgência e emergência. À época, não foram realizados nenhuma cirurgia ou procedimento invasivo, apenas medicamentos sintomáticos e antibióticos segundo o relato do paciente, porém, este não conseguiu especificar quais fármacos foram utilizados. A ultrassonografia realizada no serviço sugeriu alteração em morfologia de vesícula biliar, associada a múltiplos cálculos em seu interior, o maior medindo $1,7 \mathrm{~cm}$. Foi encaminhado ao serviço de cirurgia ambulatorial após melhora da crise álgica.

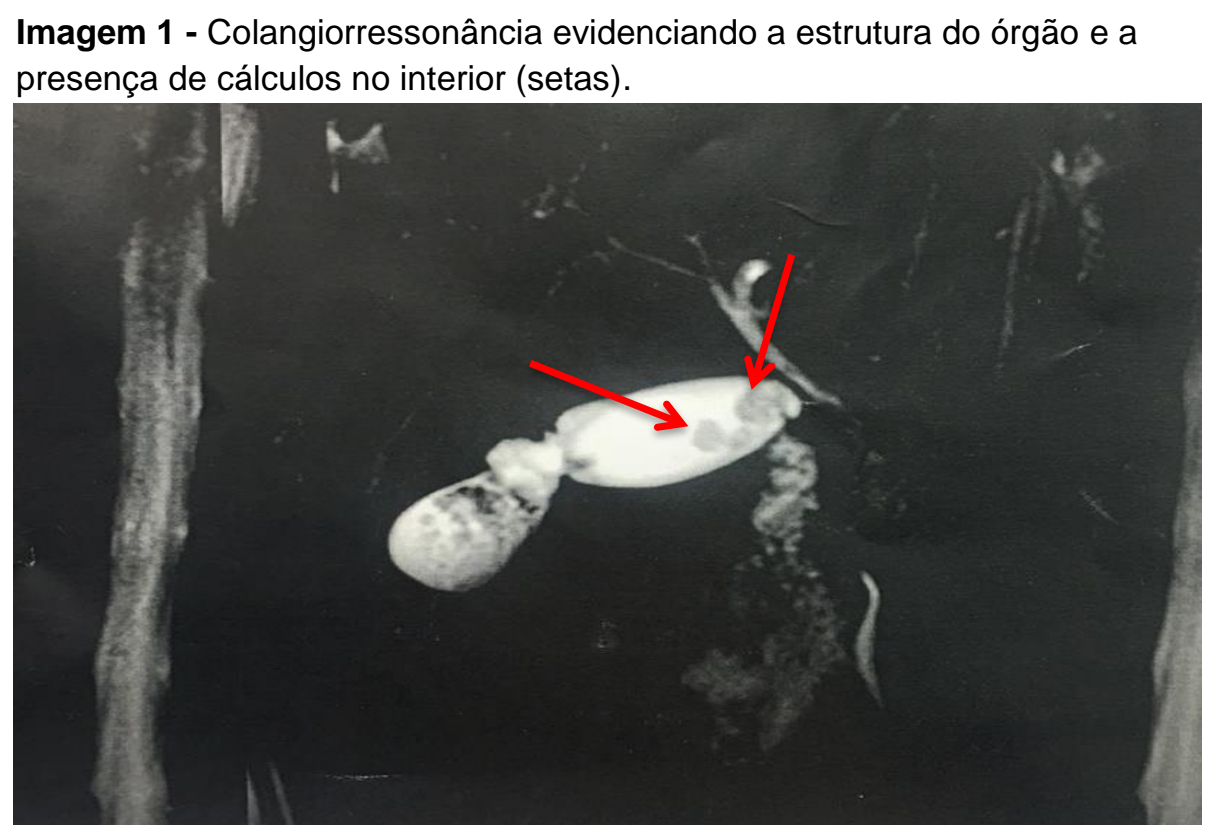

Fonte: Arquivo pessoal, 2018.

Em âmbito ambulatorial, foi constatada a necessidade de tratamento cirúrgico para colelitíase devido aos quadros sintomáticos que apresentava, mas, observada a alteração da morfologia de vesícula pelo laudo ultrassonográfico, solicitou-se colangioressonânica magnética $(\mathrm{CM})$ para exclusão de alterações anatômicas que pudessem alterar a técnica cirúrgica a ser utilizada. Em imagem de $\mathrm{CM}$, observam-se múltiplos cálculos em seu interior. Também se evidenciou bilobulação de vesícula em seu fundo, com presença calculosa no mesmo (Imagem 1). Justifica-se aqui a colecistite de longa duração há 2 anos, mas não a ausência de tratamento cirúrgico. Já em período pré-operatório, paciente apresentava-se corado, normotenso, com alteração discreta de glicemia (corrigida posteriormente ao procedimento), anictérico e sem quaisquer outras 
alterações. Apresentava dor à palpação profunda, abdômen sem visceromegalias ou abaulamentos, sem presença de sinais de irritação peritoneal. Ausência de sinal de Blumberg, mas apresentava sinal de Murphy positivo de maneira discreta.

A cirurgia realizada foi a colecistectomia videolaparoscópica, transcorrendo sem complicações durante o intraoperatório, sem dificuldades de acesso ao triângulo de Calot ou exérese de vesícula. Em visualização da peça cirúrgica, não foi possível identificar alterações notáveis em sua superfície externa (Imagem 2), sendo a mesma enviada para análise histológica assim que finalizado procedimento. Realizado o fechamento das incisões da videolaparoscopia, o paciente foi direcionado aos cuidados de recuperação pós-anestésica. Retornou para a ala cirúrgica da enfermaria, apenas com queixas leves de dor em abdome difusamente, com melhora após uso de sintomáticos. Não apresentou nenhuma queixa nas primeiras 24 horas pós-operatórias, tendo alta 36 horas após a sua recuperação anestésica.

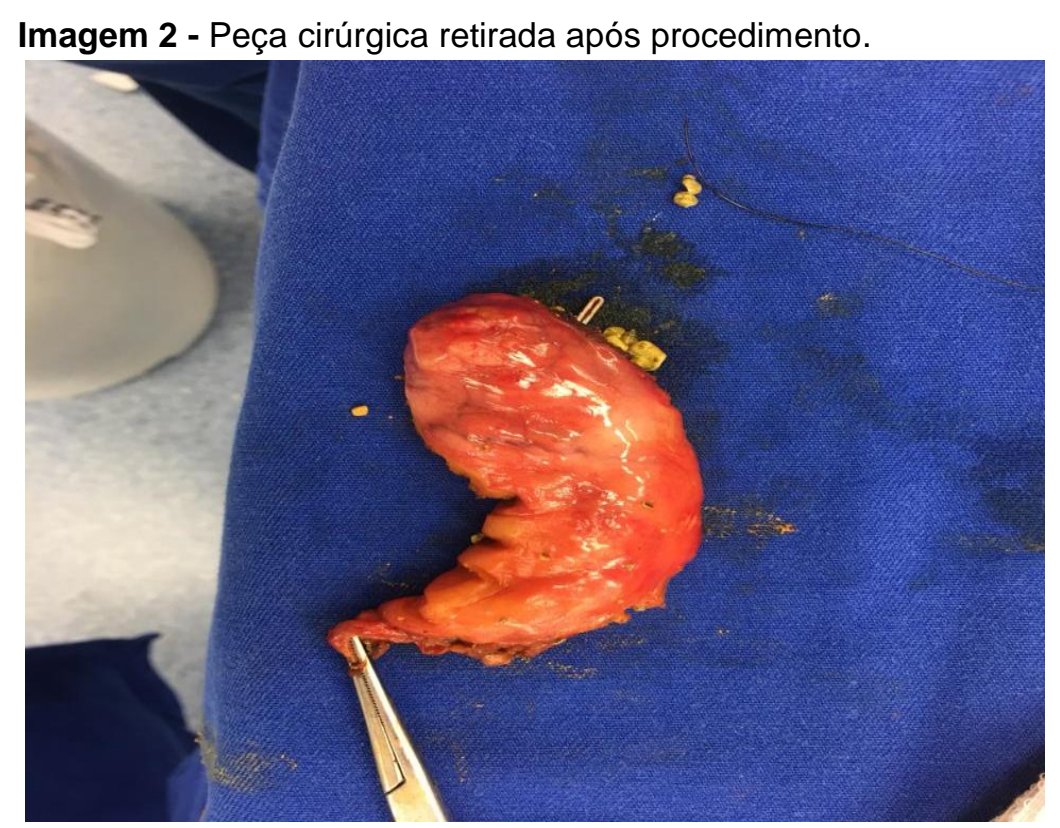

Fonte: Arquivo pessoal, 2018.

\section{DISCUSSÃO}

A duplicação da vesícula biliar na embriogênese pode ser classificada em quatro categorias: alterações de localização, de desenvolvimento, de número e de rotação (ELORZA JD et al., 2009).

Dentre as alterações de localização, a mais frequente é a vesícula biliar intra-hepática, geralmente encontrada no lobo hepático direito. A alteração de rotação gera uma vesícula que se desenvolve à esquerda do ligamento falciforme. As alterações de número se traduzem em duplicação do órgão ou agenesia deste, com está sendo de caráter extremamente raro uma vez que é encontrada em $0,03 \%$ da população. As alterações de desenvolvimento consistem na vesícula flutuante (encontrada em 10\% das pessoas), vesícula septada, vesícula com divertículos e vesícula com fundo pregueado (ELORZA JD et al., 2009).

Boyden EA (1929) descreveu um sistema de classificação de duplicações da vesícula biliar, uma anomalia de número. Segundo essa classificação, a vesícula pode apresentar bilobulação/Vesica fellea divisa (que tem um único ducto cístico) ou duplicação verdadeira/Vesica fellea duplex, a qual é subclassificada em "tipo Y" (dois ductos císticos que se unem antes de entrar no ducto biliar comum) e "em forma de H ou tipo ductular" (dois ductos císticos que entram separadamente no ducto biliar comum).

Harlaftis $N$ et al. (1977) modificaram esta classificação, descrevendo essa variação em dois grupos principais, tipo I e tipo II, sendo essa a classificação mais adotada hoje. O tipo I consiste em vesículas com 
as seguintes características: divisão parcial por um septo, divisão incompleta em forma de $V$ com ambas os "lóbulos" unidas no colo e divisão quase completa em Y, apresentando dois ductos císticos unidos em um ducto cístico comum responsável por fazer a drenagem ao ducto biliar comum.

No tipo II, as anomalias mais comuns são as vesículas biliares acessórias (presença de duas vesículas biliares separadas, sejam elas dotadas de ductos císticos independentes e que drenam direta e individualmente seu conteúdo no ducto biliar comum, conhecido como subtipo $\mathrm{H}$, ou com um dos ductos císticos drenando para o ducto hepático direito ou esquerdo, conhecido como subtipo trabecular) (HARLAFTIS $\mathrm{N}$ et al., 1977).

O paciente em questão apresentava vesícula do tipo Vesica fellea divisa, segundo a classificação original de Boyden ou vesícula do tipo I, de acordo com a classificação de Boyden EA (1929) modificada por Harlaftis $\mathrm{N}$ et al. (1977). A diferenciação sintomatológica de um subtipo anatômico para outro, assim como a distinção entre um caso de colelitíase em vesícula única ou em vesícula duplicada é impraticável já que não há divergência nos sinais e sintomas clínicos entre os casos.

A identificação da presença de vesícula biliar bilobulada/ duplicada se dá a partir da realização de exames de imagem, em especial a ultrassonografia, permitindo que o cirurgião planeje o procedimento de modo mais seguro. Nos casos de colelitíase, a conduta mais efetiva é a retirada total do órgão, de modo a evitar reoperações (DESOULNEUX G et al., 2009).

\section{REFERÊNCIAS}

1. BOYDEN EA. The accessory gall-bladder: an embryological and comparative study of aberrant biliary vesicles occurring in man and the domestic mammals. American Journal of Anatomy Banner, 1926; 38 (2):177-231.

2. COELHO JCU, et al. Colecistectomia laparoscópica em um paciente com ducto cístico duplo. Revista do Colégio Brasileiro de Cirurgiões, 2003; 30: 486-8.

3. DARWISH HS. Duplication of Gall bladder: Review of Literature and Report of a Case. OMICS Journal of Radiology, 2016.

4. DESOLNEUX G, et al. Duplication of the Gallbladder. A Case Report. Gastroenterology Research and Practice, 2009.

5. ELORZA JD, et al. Duplicación de la vesícula biliar: Vesícula doble de tipo Y operada. Reporte de un caso. Revista Chilena de Cirugía, 2009; 61 (3): 275-278.

6. GOCMEN R, YESILKAYA Y. Imaging findings of gallbladder duplication due to two cases: case report and review of literature. Medical Ultrasonography, 2012; 14 (4): 358-360

7. HARLAFTIS N, et al. Multiple gallbladders. Surgery, Gynecology \& Obstetrics, 1977; 145 (6): 928-934.

8. HEKIMOGLU K, et al. Combined use of ultrasonography, MDCT and MRCP for the diagnosis of gallbladder duplication: case report. Journal of Digestive Diseases, 2010; 11: 115 -118.

9. LOBO EJ, et al. Laparoscopic cholecystectomy in a patient with duplicated cystic duct. Surgical Laparoscopy, Endoscopy \& Percutaneous Techniques, 2000; 10: 326-328.

10. MADDOX JM, DEMERS ML. Laparoscopic management of gallbladder duplication: a case report and review of literature. Journal of the Society of Laparoendoscopic Surgeons, 1999; 3: 137-140.

11. PINA LF, et al. Vesícula biliar duplicada com abordagem laparoscópica: relato de caso. ABCDExpress, 2018.

12. PILLAY Y. Gallbladder duplication. International Journal of Surgery Case Reports, 2015; 11:18-20.

13. OZGEN A, et al. Gallbladder duplication: imaging findings and differential considerations. Abdominal Imaging, 1999; 24: $285-288$.

14. ROMERO EXA, et al. Gallbladder duplication and cholecystitis. Journal of Surgical Case Reports, 2018; 2018 (7).

15. SAFIOLEAS MC, et al. Congenital duplication of the gallbladder. The American Surgeon, 2006; 72: 217- 220.

16. SHIBA H, et al. Duplicated Gallbladder. International Surgery, 2014; 99 (1): 77 - 18.

17. SINGH B, et al. Duplicate gallbladder: an unusual case report. Surgical and Radiologic Anatomy, 2006; 28: 654- 657. 